The Supercontinuum Laser Source 
Robert R. Alfano

Editor

\section{The Supercontinuum Laser Source}

With 250 Illustrations

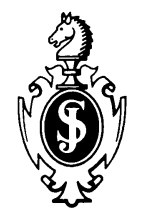

Springer Science+Business Media, LLC 
Robert R. Alfano

Distinguished Professor of Science and Engineering

Institute for Ultrafast Spectroscopy and Lasers

Physics and Electrical Engineering Departments

The City College of New York

New York, NY 10031, USA

Library of Congress Cataloging-in-Publication Data

The Supercontinuum laser source/Robert R. Alfano, editor.

p. $\mathrm{cm}$.

Includes bibliographies and index.

Contents: Theory of self-phase modulation and spectral broadening

/ Y.R. Shen, Guo-Zhen Yang-Supercontinuum generation in condensed matter / Q.Z. Wang, P.P. Ho, R.R. Alfano-Ultrashort pulse propagation in nonlinear dispersive fibers / Govind P. AgrawalCross-phase modulation: a new technique to control the spectral, temporal, and spatial properties of ultrashort pulses / P.L.

Baldeck, P.P. Ho, R.R. Alfano-Simple models of self-phase and induced-phase modulation / Jamal T. Manassah-Self steepening of optical pulses / B.R. Suydam-Self-focusing and continuum generation in gases / Paul B. Corkum and Claude Rolland-The utilization of UV and IR supercontinua in gas-phase subpicosecond kinetic spectroscopy / J.H. Glownia, J. Misewich, P.P. SorokinApplications of supercontinuum / R. Dorsinville, P.P. Ho, J. Manassah, R.R. Alfano-Pulse compression and femtosecond continuum/

C. Shank and A. Johnson.

1. Laser pulses, Ultrashort. 2. Nonlinear optics. I. Alfano. R.R. QC689.5.L37S87 1989

621.36'6-dc19

Printed on acid-free paper

Cover shows supercontinuum generation of intensity vs. wavelength for $1 \mathrm{~mm}$ of carbon tetrachloride liquid excited by a $120-\mathrm{fs}, 625-\mathrm{nm}$ laser pulse.

Photo by R. Alfano, A. Katz, and P. Ho.

(C) 1989 by Springer Science+Business Media New York

Originally published by Springer-Verlag New York Inc. in 1989

Softcover reprint of the hardcover 1st edition 1989

All rights reserved. This work may not be translated or copied in whole or in part without the written permission of the publisher Springer Science+Business Media, LLC,

except for brief excerpts in connection with reviews or scholarly analysis. Use in connection

with any form of information storage and retrieval, electronic adaptation, computer software, or by similar or dissimilar methodology now known or hereafter developed is forbidden.

The use of general descriptive names, trade names, trademarks, etc. in this publication, even if the former are not especially identified, is not to be taken as a sign that such names, as understood by the Trade Marks and Merchandise Marks Act, may accordingly be used freely by anyone.

Typeset by Asco Trade Typesetting Ltd., Hong Kong. 
To my father, Alfonso L. Alfano

and my father-in-law, Samuel J. Resnick

whose advice I deeply miss. 


\section{Preface}

This book deals with both ultrafast laser and nonlinear optics technologies. Over the past two decades, we have seen dramatic advances in the generation of ultrafast laser pulses and their applications to the study of phenomena in a variety of fields. It is now commonplace to produce picosecond $\left(10^{-12} \mathrm{~s}\right)$ pulses. New developments have extended this technology into the femtosecond $\left(10^{-15} \mathrm{~s}\right)$ time region. Soon pulses consisting of just a single cycle will be produced (i.e., $2 \mathrm{fs}$ at $600 \mathrm{~nm}$ ). These ultrafast pulses permit novel investigations to study phenomena in many disciplines. Sophisticated techniques based on these laser pulses have given rise to instruments with extremely high temporal resolution. Ultrafast laser technology offers the possibility of studying and discovering key processes unresolved in the past. A new era of time-resolved spectroscopy has emerged, with pulses so fast that one can now study the nonequilibrium states of matter, test quantum and light models, and explore new frontiers in science and technology. Ultrashort light pulses are a potential signal source in future high-bit-rate optical fiber communication systems. The shorter the pulses, the more can be packed into a given time interval and the higher is the data transmission rate for the tremendous band width capacity of optical fiber transmission.

Nonlinear optics is an important field of science and engineering because it can generate, transmit, and control the spectrum of laser pulses in solids, liquids, gases, and fibers. One of the most important ultrafast nonlinear optical processes is the supercontinuum generation - the production of intense ultrafast broadband "white light" pulses - that is the subject of this book.

The first study on the mechanism and generation of ultrafast supercontinuum dates back over 19 years to 1969, when Alfano and Shapiro observed the first "white" picosecond pulse continuum in liquids and solids. Spectra extended over $\sim 6000 \mathrm{~cm}^{-1}$ in the visible and infrared wavelength region. They attributed the large spectral broadening of ultrafast pulses to self-phase modulation (SPM) arising from an electronic mechanism and laid down the formulation of the supercontinuum generation model. Over the years, the improvement of mode-locked lasers led to the production of wider super- 
continua in the visible, ultraviolet, and infrared wavelength regions using various materials.

The supercontinuum arises from the propagation of intense picosecond or shorter laser pulses through condensed or gaseous media. Various processes are responsible for continuum generation. These are called self-, induced-, and cross-phase modulations and four-photon parametric generation. Whenever an intense laser pulse propagates through a medium, it changes the refractive index, which in turn changes the phase, amplitude, and frequency of the pulse. However, when two laser pulses of different wavelengths propagate simultaneously in a condensed medium, coupled interactions (cross-phase modulation and gain) occur through the nonlinear susceptibility coefficients. These coupled interactions of two different wavelengths can introduce phase modulation, amplitude modulation, and spectral broadening in each pulse due to the other pulse using cross-effects.

An alternative coherent light source to the free electron laser, the supercontinuum laser source, can be wavelength selected and coded simultaneously over wide spectral ranges (up to $10,000 \mathrm{~cm}^{-1}$ ) in the ultraviolet, visible, and infrared regions at high repetition rates, gigawatt output peak powers, and femtosecond pulse durations.

Ultrafast supercontinuum pulses have been used for time-resolved absorption spectroscopy and material characterization. Supercontinuum generation is a key step for the pulse compression technique, which is used to produce the shortest optical pulses. Future applications include signal processing, three-dimensional imaging, ranging, atmospheric remote sensing, and medical diagnosis.

Thus far, a great deal of information on supercontinuum technology has been obtained and has enhanced our understanding of how intense optical pulses propagate in materials. These developments are most often found in original research contributions and in review articles scattered in journals. Textbooks do not cover these subjects in great detail. There is a need for a book that covers the various aspects of ultrafast supercontinuum phenomena and technology.

This book reviews present and past progress on the experimental and theoretical understanding of ultrafast nonlinear processes responsible for supercontinuum generation and related effects such as pulse compression and ultrashort pulse generation on a picosecond and femtosecond time scale. The content of the chapters in the book is a mixture of both theoretical and experimental material. Overviews of the important breakthroughs and developments in the understanding of supercontinuum during the past 20 years are presented. The book is organized into 10 chapters.

Summarizing the highlights of the 10 chapters of the book:

In Chapter 1, Shen and Yang focus on the theoretical models and mechanisms behind supercontinuum generation arising mainly from self-phase modulation. 
In Chapter 2, Wang, Ho, and Alfano review the experiments leading to the supercontinuum generation in condensed matter over the past 20 years.

In Chapter 3, Agrawal discusses the effects of dispersion on ultrafast light pulse propagation and supercontinuum generation in fibers.

In Chapter 4, Baldeck, Ho, and Alfano cover the latest experimental observations and applications of the cross-interactions in the frequency, time, and space domains of strong pulses on weak pulses.

In Chapter 5, Manassah reviews the theoretical models giving rise to many phenomena from self-phase and induced modulations.

In Chapter 6, Suydam highlights the effect of self-steepening of pulse profile on continuum generation.

In Chapter 7, Corkum and Rolland review the work on supercontinuum and self-focusing in gaseous media.

In Chapter 8, Glownia, Misewich, and Sorokin utilize the supercontinuum produced in gases for ultrafast spectroscopy in chemistry.

In Chapter 9, Dorsinville, Ho, Manassah, and Alfano cover the present and speculate on the possible future applications of the supercontinuum in various fields.

In Chapter 10, Johnson and Shank discuss pulse compression from the picosecond to femtosecond time domain using the continuum and optical dispersive effects of gratings, prisms, and materials.

The reader will find that these chapters review the basic principles, contain surveys of research results, and present the current thinking of experts in the supercontinuum field. The volume should be a useful source book and give young and seasoned scientists, engineers, and graduate students an opportunity to find the most necessary and relevant material on supercontinuum technology in one location.

I hope these efforts will stimulate future research on understanding the physics behind supercontinuum technology and exploring new applications.

I wish to thank all the expert contributors for their cooperation in this endeavor. Most thought it would not be completed. Special thanks goes to Mrs. Megan Gibbs for her administrative and secretarial assistance. I gratefully acknowledge T. Hiruma for his continued support. I pay particular tribute to my friend Stan Shapiro, who missed seeing the outgrowth of our first work in this field 20 years ago. 


\section{Contents}

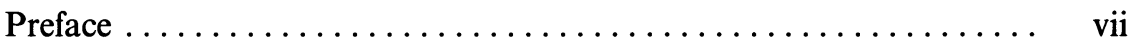

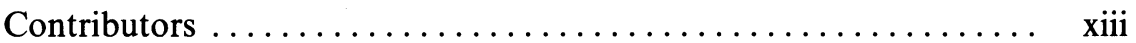

Chapter 1 Theory of Self-Phase Modulation and Spectral Broadening

Y.R. SHEN and GUO-ZHEN YANG . . . . . . . . . . . 1

Chapter 2 Supercontinuum Generation in Condensed Matter

Q.Z. WANG, P.P. Ho, and R.R. AlfaNo ......... 33

Chapter 3 Ultrashort Pulse Propagation in Nonlinear

Dispersive Fibers

Govind P. AGRAWAL

Chapter 4 Cross-Phase Modulation: A New Technique for

Controlling the Spectral, Temporal, and Spatial

Properties of Ultrashort Pulses

P.L. Baldeck, P.P. Ho, and R.R. Alfano ......... 117

Chapter 5 Simple Models of Self-Phase and

Induced-Phase Modulation

Jamal T. MANASSAH .......................... 184

Chapter 6 Self-steepening of Optical Pulses

B.R. SUYDAM ......................... 295

Chapter 7 Self-focusing and Continuum Generation in Gases

Paul B. Corkum and Claude Rolland ........... 318

Chapter 8 Utilization of UV and IR Supercontinua in Gas-Phase Subpicosecond Kinetic Spectroscopy

J.H. Glownia, J. Misewich, and P.P. SOROKIN . . . . . . 337 
xii Contents

Chapter 9 Applications of Supercontinuum: Present and Future

R. Dorsinville, P.P. Ho, Jamal T. Manassah, and

R.R. Alfano . . . . . . . . . . . . . . . . . . . . . . 377

Chapter 10 Pulse Compression in Single-Mode Fibers-Picoseconds to Femtoseconds

A.M. Johnson and C.V. SHANK .............. 399

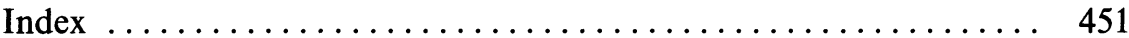




\section{Contributors}

Govind P. AGRaWaL, Institute of Optics, University of Rochester, Rochester, New York 14627, USA

R.R. Alfano, Departments of Physics and Electrical Engineering, City College of the City University of New York, New York 10031, USA

P.L. BALDECK, Department of Electrical Engineering, City College of the City University of New York, New York 10031, USA

Paul B. Corkum, Division of Physics, National Research Council of Canada, Ottawa, Ontario, K1A OR6 Canada

R. Dorsinville, Department of Electrical Engineering, City College of the City University of New York, New York 10031, USA

J.H. GLOWNIA, IBM Research Division, Thomas J. Watson Research Center, Yorktown Heights, New York 10598, USA

P.P. Ho, Department of Electrical Engineering, City College of the City University of New York, New York 10031, USA

A.M. Johnson, AT\&T Bell Laboratories, Holmdel, New Jersey 07733, USA

Jamal T. Manassah, Department of Electrical Engineering, City College of the City University of New York, New York 10031, USA

J. MisewICH, IBM Research Division, Thomas J. Watson Research Center, Yorktown Heights, New York 10598, USA

Claude Rolland, Division of Physics, National Research Council of Canada, Ottawa, Ontario, K1A OR6 Canada 
C.V. Shank, AT\&T Bell Laboratories, Holmdel, New Jersey 07733, USA

Y.R. SHEN, Department of Physics, University of California, Berkeley, California 94720, USA

P.P. SoROKIN, IBM Research Division, Thomas J. Watson Research Center, Yorktown Heights, New York 10598, USA

B.R. SuYDam, Los Alamos National Laboratory, Los Alamos, New Mexico 87545, USA

Q.Z. WANG, Department of Physics, City College of the City University of New York, New York 10031, USA

Guo-Zhen YANG, Institute of Physics, Academy of Sciences, Beijing, China 